\title{
Introducción a la irradiación gamma de alimentos y desarrollo tecnológico en Colombia
}

\section{Introduction to gamma irradiation of food and technological development in Colombia}

Jormagn Israel Abril ${ }^{1}$

Citación: J. I. Abril, "Introducción a la irradiación gamma de alimentos y desarrollo tecnológico en Colombia”, Revista de Investigaciones y Aplicaciones Nucleares, n. ${ }^{\circ}$ 2, pp. 5-14, 2018.

Recibido: 2 de julio de 2018

Aceptado: 9 de octubre de 2018

Publicado en línea: 17 de

diciembre de 2018

Doi: 10.32685/2590-7468/invapnuclear.2.2018.49

Palabras clave:

Irradiación gamma de alimentos, conservación de alimentos, inocuidad, calidad, investigación y desarrollo.

Keywords: Gamma irradiation of foods, food preservation, safety, quality, research and development.

\section{RESUMEN}

La irradiación gamma es un método seguro y efectivo de control de microorganismos dañinos y patógenos en diferentes clases de productos que los humanos consumen en todo el mundo, sean alimentos o productos de farmacia, cosmética y dispositivos médicos. La irradiación gamma de alimentos se utiliza principalmente con el fin de lograr su preservación mediante la reducción del deterioro, el mejoramiento de su higiene y el aumento de su vida útil. Son varias las aplicaciones en la industria alimentaria, que van desde desinfestación de frutas y semillas, inhibición de brotes en tubérculos, conservación y desinfección, hasta esterilización, de todas las cuales derivan múltiples beneficios; cada una suple una necesidad que otras tecnologías no satisfacen totalmente, lo cual genera un menor impacto económico y ambiental. A pesar de su importante aporte a la preservación e inocuidad de los alimentos, en el ámbito nacional no se aplica la tecnología de irradiación en las cadenas de producción y se desconocen los efectos específicos de la técnica sobre la calidad de los productos locales, así como las características inherentes de posibles productos irradiados que entran al país, a lo cual se suma una desconsiderada falta de regulación que respalde su explotación segura.

\section{ABStraCt}

Gamma irradiation is a safe and effective method for the control of harmful microorganisms and pathogens in different kinds of products for human consumption around the world, in food, pharmacy, cosmetics and medical devices. Gamma irradiation of food is mainly used in order to achieve its preservation through the reduction of deterioration, improvement of its hygiene and increase in useful life. There are several applications in the food industry, ranging from disinfestation of fruits and seeds, inhibition of tuber sprouts, conservation, disinfection to sterilization, all with multiple benefits; each one supplies a need that other technologies do not fully satisfy, generating a lower economic and environmental impact. Despite its important contribution to the preservation and safety of food, at national level, radiation technology is not applied in the production chains and the specific effects of the technique on the quality of local products are unknown, as well as the inherent characteristics of possible irradiated products that enter the country, added to a inconsiderate lack of regulation that supports their exploitation in a safe way.

\footnotetext{
${ }^{1}$ Ingeniero químico, especialista en ciencia y tecnología de alimentos. Servicio Geológico Colombiano Email de correspondencia: jabril@sgc.gov.co
} 


\section{INTRODUCCIÓN}

E 1 uso de radiación gamma en alimentos ha demostrado su efectividad en los tratamientos para conservación, desinfestación de plagas cuarentenarias y en reducción de cargas microbianas, tanto patógenas como las que simplemente causan deterioro en los alimentos [1]-[4], pues aumentan su vida útil, calidad e inocuidad [1]. También denominada "pasteurización en frío", esta técnica radiactiva es considerada un punto crítico de control y se usa ampliamente en los sectores de alimentos procesados y en aquellos que se comercializan o se distribuyen crudos o poco procesados (carnes, pescado, aves, frutas y verduras) a nivel mundial [5]. La utilización de radiación gamma proveniente de una fuente de cobalto-60 $\left({ }^{60} \mathrm{Co}\right.$ ) (isótopo radiactivo del ${ }^{59} \mathrm{Co}$ original) en alimentos ha sido tema de controversia debido a que el consumidor no es consciente de la aplicación de tal procedimiento; a pesar de ello, ha disminuido la percepción negativa y entre cuarenta y cincuenta países han aprobado su uso [6], [7].

La razón principal que justifica el uso de radiación ionizante como tratamiento de alimentos es la misma que justifica cualquier otro uso con fuentes radiactivas: un ahorro significativo de tiempo y la disminución de esfuerzo [8]; adicionalmente, encontramos que la relación de costo-beneficio es considerablemente mayor en comparación con otras tecnologías de conservación [9]. Diversos autores coinciden en que son más los beneficios que los perjuicios implicados en el tratamiento correcto de alimentos con radiación gamma [9], [10].

Después del descubrimiento de la radiación ionizante en 1896 por Röentgen y Becquerel, así como de sus efectos sobre la materia, surgieron especulaciones sobre cómo se podría aplicar este fenómeno, tanto en el tratamiento terapéutico (Minck) como en la conservación de alimentos aprovechando su característico efecto bactericida (Samuel Prescott, MIT, 1904, [11]). La pregunta de cómo aplicar dicha tecnología llevó a considerar ideas tan escabrosas como la propuesta de mezclar alimentos con sustancias radiactivas (Lieber, patent, 1905). Debido a que no se contaba aún con fuentes radiactivas adecuadas para la explotación a mayor escala, se partió de los desarrollos militares (Guerra Fría), con lo cual se contó con fuentes selladas, en especial fuentes de cesio-137 $\left({ }^{137} \mathrm{Cs}\right)$ que comenzaron a estar disponibles; incluso el combustible nuclear gastado se consideró como una fuente de irradiación. No fue sino hasta la disponibilidad de reactores de potencia, y con la producción de radioisótopos, que el ${ }^{60} \mathrm{Co}$ se convirtió en un estándar industrial [11], y ya en 1964 la compañía de tecnología nuclear canadiense Nordion instaló en el irradiador de almacenamiento en húmedo de la ciudad de Sommerville, NJ, las primeras fuentes selladas de ${ }^{60} \mathrm{Co}$, denominadas ${ }^{188} \mathrm{C}$, las cuales permitían un diseño modular del irradiador, fácil de manipular como de transportar [7].

Desde mediados del siglo XX, Europa fue pionera en el estudio y la explotación segura de la radiación ionizante con diferentes fines, siendo Alemania el primer país donde se instaló, entre 1957 y 1958, en la ciudad de Stuttgart, una planta de irradiación de especies, que era un acelerador de electrones [11], [12]. Sin embargo, son los países europeos los que menos están utilizando la tecnología, y en especial en alimentos [10], [12], debido a la baja aceptación de los consumidores, pero encuestas realizadas en otros países, como por ejemplo EE. UU., demuestran que dicho inconveniente es un problema de desinformación, más cuando contrastan las ventajas de la radiación con el uso de agroquímicos o los tratamientos térmicos convencionales [10].

Basado en estadísticas relativas al período comprendido entre el 2005 y el 2010, Peter B. Roberts [10] encontró que la irradiación de alimentos disminuyó en Europa y aumentó en algunas partes de Asia y en los EE. UU., Australasia y otras regiones. También reportó que los datos de 2010 dan un total global de alimentos irradiados de aproximadamente 400000 t. Suponiendo que es una subestimación, el mismo autor considera que un millón de $t$ al año es un valor más adecuado, pues, para el año 2013 había aumentado significativamente, para un total de $700000 \mathrm{t}$, dato reportado por el OIEA en su Manual para las buenas prácticas de irradiación de alimentos, publicado el 2015 [13]. Aun así, estas cifras son solo una pequeña fracción de la producción y consumo global de alimentos.

La calidad e inocuidad alimentaria se ha vuelto un asunto de importancia en países en vías de desarrollo, donde la irradiación se presenta como una solución alternativa a nuevos productos que abastecen mercados locales y regionales. Países de Asia, Australasia, África, Centroamérica y Sudamérica están avanzando en el conocimiento y la aplicación de nuevas tecnologías que aseguren el suministro de alimentos y reduzcan las pérdidas por temporada de diferentes productos. 
En Colombia, a pesar de que cuenta con una planta de irradiación demostrativa a escala piloto, dotada con fuentes de cobalto desde mediados de los años setenta [14], no se ha transferido la tecnología al sector privado ni se ha constituido una legislación sólida que respalde a las instituciones que controlan el comercio de alimentos, como el Invima, o vigilan el tratamiento de frutos para exportación por control cuarentenario o control de plagas, como el ICA, o incluso el Ministerio de Cultura, una de cuyas funciones es la protección del patrimonio cultural -otra aplicación entre muchas de la radiación ionizante- En perspectiva, mediante este escrito se intenta hacer conocer a las partes interesadas de las cadenas de producción de alimentos que funcionan en el país, las ventajas y condiciones relativas a la explotación de esta tecnología, por qué no, a escala industrial.

\section{EFECTO DE LA IRRADIACIÓN GAMMA EN ALIMENTOS: ¿POR QUÉ ES SEGURO CONSUMIR ALIMENTOS IRRADIADOS?}

La tecnología de irradiación gamma ionizante reduce la carga microbiana patógena causante de enfermedades como la producida por la bacteria E. Coli, aumenta los tiempos de vida en anaquel por reducción de hongos y levaduras [4] que deterioran los productos y es un método de control contra insectos exóticos provenientes del comercio internacional, entre otras muchas ventajas, lo que hace de esta aplicación un tratamiento con múltiples fines, dependiendo de la dosis aplicada.

El consumo seguro de alimentos irradiados, debido a su inocuidad, ha sido documentado en estudios de cooperación internacional y reconocido por la Organización de las Naciones Unidas para la Alimentación y la Agricultura (FAO), el Comité sobre Alimentos de la Comisión Europea, el Organismo Internacional de Energía Atómica (OIEA) y la Organización Mundial de la Salud (OMS), entre otras organizaciones, que han concluido que los alimentos irradiados correctamente son seguros y nutricionalmente adecuados [5], [12], lo que les permite competir comercial y sensorialmente con sus homólogos no tratados, o tratados con otras tecnologías [15].

Existen, como en todo el campo de la tecnología nuclear, miedos infundados respecto a la posibilidad de consumir sustancias radiactivas o productos contaminados pro- venientes del tratamiento con radiación. La tecnología de radiación ionizante aplicada en alimentos, junto con la de esterilización de dispositivos de uso médico, a mediados del siglo pasado llegó a un punto en que se pudo demostrar que no hay peligro alguno en consumir alimentos irradiados. Este concepto está soportado especialmente en estudios de instituciones pagadas por los Estados interesados en el uso pacífico de la tecnología nuclear, como la Escuela de Hungría, que fue liderada por el profesor Josef Farkas [16]. Para Christopher Sommers, coautor del libro Food Irradiation: Research and Technology, y otros autores, la técnica de irradiación ha sido el tratamiento de conservación de alimentos más estudiado en la historia [6], [17].

Las razones son que no se han identificado vínculos posibles con riesgos para la salud y no es cierto que la radiación pueda inducir una radiactividad artificial en dichos productos o su empaque [11], [12], ni siquiera con energías de hasta $10 \mathrm{MeV}$, que sobrepasan la energía del isótopo del cobalto, altamente utilizado, y que solo llega hasta 1,3 MeV [10], [11]. Claro está que en aceleradores de electrones es posible conseguir energías cercanas o superiores a dicho rango; aun así, estos niveles de energía no representan un riesgo para la salud [11]. Tampoco existe riesgo de contaminación del alimento, dado que las utilizadas son fuentes selladas herméticamente en cápsulas de acero inoxidable, y jamás entran en contacto con el alimento [18].

\section{3. ¿QUÉ SUCEDE CUANDO SE IRRADIA UN ALIMENTO? SUSTANCIAS QUÍMICAS PRODUCTO DE LA RADIACIÓN}

Teniendo en cuenta que el fenómeno de radiación ionizante sobre el alimento ocurre solo a nivel de las capas electrónicas del átomo, los alimentos se comportan de forma similar a cualquier otra sustancia biológicamente activa, sean seres vivos o productos orgánicos inanimados, como los alimentos, que poseen contenidos determinados de moléculas con valor nutricional, y que pueden verse afectados por la aplicación de diferentes tipos de tratamientos para conservación, descontaminación o desinfestación, entre otros. Dichos efectos, que dependen del grado o cantidad del agente utilizado para el tratamiento, sea térmico o por el uso de sustancias químicas $o$ aditivos, pueden causar deterioro del alimento si son suministrados en exceso. Igual ocurre con la radiación ionizante 
(que tiene efectos similares a los del calentamiento térmico [19] en grasas y aceites, por cuanto produce oxidación de las cadenas lipídicas [20]) y otros procesos térmicos, como el enlatado [17] y el secado [21].
A continuación, se presenta una tabla en la que se relacionan algunos de los tipos de tratamiento o aditivos utilizados, y las principales sustancias perjudiciales encontradas en los alimentos después de ser tratados, así como los efectos que en la salud tiene el consumo de dichos alimentos:

Tabla 1. Tratamientos convencionales y uso de aditivos en alimentos para alcanzar fines de conservación y desinfección bacteriana

\begin{tabular}{|c|c|c|c|c|}
\hline Tipo de tratamiento & Alimento & Sustancias químicas producidas & Efectos en la salud & Referencias \\
\hline $\begin{array}{l}\text { Cocción y curado, } \\
\text { más uso de nitritos }\end{array}$ & Carnes de res y su grasa & Nitrosaminas & Mutagénico & {$[20]$} \\
\hline Cocción y curado & Carne de res, aves y su grasa & Productos de oxidación & Carcinógeno & {$[20]$} \\
\hline Frituras y horneado & $\begin{array}{l}\text { Productos con contenido } \\
\text { de almidón }\end{array}$ & Acrilamida & Carcinógeno & {$[20]$} \\
\hline Procesos térmicos & Alimentos & Furanos & Carcinógeno & {$[20]$} \\
\hline Cocción & Lípidos & $\begin{array}{l}\text { Compuestos ciclados (benzo[a] } \\
\text { pireno e Imidazoquinolina) }\end{array}$ & Carcinógeno & {$[21]$} \\
\hline $\begin{array}{l}\text { Inclusión de aditivos } \\
\text { bactericidas }\end{array}$ & Embutidos & $\begin{array}{l}\text { Benzoatos, sorbatos, } \\
\text { propionatos, nitritos, nitratos, } \\
\text { sulfitos y parabenos }\end{array}$ & Mutagénicos, carcinógeno & {$[22]$} \\
\hline
\end{tabular}

Fuente: autor

En el contexto de la irradiación gamma de alimentos, los riesgos asociados son similares a los de otros procesos. El deterioro de la calidad producido por la formación de moléculas propias de la irradiación ha sido ampliamente estudiado, y ya han sido identificados y clasificados los productos de la radiólisis de cada componente de los alimentos (grasas, minerales, vitaminas, proteínas y aminoácidos, carbohidratos, etc.). Se sabe qué dosis máxima absorbida puede tener efectos negativos en los nutrientes, a un rango de detección determinado, y qué límites son perjudiciales para la salud humana cuando los productos así tratados son consumidos [17], [20].

En 1995 la Organización Mundial de la Salud publicó un libro que recopila sistemáticamente todo lo relacionado con la técnica, titulado Inocuidad e idoneidad nutricional de los alimentos irradiados [21], que puede descargarse gratis en la página de la OMS dedicada a ampliar el conocimiento del lector (http://apps.who.int/iris/handle/10665/37226). De especial interés es el capítulo 6, al final del cual se concluye que no hay indicios que demuestren que los compuestos producidos por la radiación, en las concentraciones que aparecen a elevadas dosis (entre 25 y $60 \mathrm{kGy}$ ), entrañe riesgo toxicológico alguno [21], tanto así que se encuentran mayores subproductos al tostar pan o al asar carne [17]. A continuación se resumen los principales compuestos, el componente alimenticio relacionado, así como una comparación con los tratamientos convencionales; cabe anotar que incluso con dosis de radiación tan altas como 50 kGy se necesitaron varios años de desarrollo para mejorar el nivel de detección de los métodos de análisis que pueden identificar dichos compuestos [21]:

Tabla 2. Productos de irradiación de los alimentos identificados por componente principal

\begin{tabular}{|c|c|c|c|}
\hline Componente del alimento & $\begin{array}{l}\text { Compuesto producto } \\
\text { de la irradiación }\end{array}$ & $\begin{array}{l}\text { Tratamiento convencional que produce } \\
\text { un efecto análogo }\end{array}$ & Referencia \\
\hline Grasa animal (carne de res) & Benceno & $\begin{array}{l}\text { - Descomposición } \\
\text { del benzoato de potasio } \\
\text { - Cocción }\end{array}$ & {$[20]$} \\
\hline Carbohidratos & $\begin{array}{l}\text { Formaldehído, y en solución acuosa, } \\
\text { ácidos, alcoholes, peróxidos aldehídos } \\
\text { y cetonas }\end{array}$ & Cocción & {$[20],[21]$} \\
\hline Carbohidratos & $\begin{array}{l}\text { Polisacáridos como dextrinas, maltosa } \\
\text { y glucosa }\end{array}$ & Cocción & {$[21]$} \\
\hline Lípidos & $\begin{array}{l}\text { Hidrocarburos, ésteres, aldehídos y } \\
\text { cetonas }\end{array}$ & Cocción & {$[20]$} \\
\hline Lípidos & $\begin{array}{l}\text { 2-alkilciclobutanonas } \\
\text { (2-ACB) }\end{array}$ & Ninguno & {$[20]$} \\
\hline
\end{tabular}




\begin{tabular}{|c|c|c|c|}
\hline Componente del alimento & $\begin{array}{l}\text { Compuesto producto } \\
\text { de la irradiación }\end{array}$ & $\begin{array}{l}\text { Tratamiento convencional que produce } \\
\text { un efecto análogo }\end{array}$ & Referencia \\
\hline Proteínas & $\begin{array}{l}\text { Polipéptidos de menor tamaño o } \\
\text { agregaciones entre proteínas }\end{array}$ & Cocción & {$[21]$} \\
\hline Enzimas & $\begin{array}{l}\text { No sufren modificación, y solo el calor } \\
\text { puede inactivarlasz }\end{array}$ & No es aplicable & {$[21]$} \\
\hline Vitaminas (aisladas en solución) & $\begin{array}{l}\text { Pérdida de la vitamina } \mathrm{B} 1 \text { en alrededor } \\
\text { de un } 50 \% \text { con } 0,5 \mathrm{kGy}\end{array}$ & No es aplicable & {$[21]$} \\
\hline $\begin{array}{l}\text { Vitaminas (Contenidas en la matriz real } \\
\text { del alimento) }\end{array}$ & $\begin{array}{l}\text { - Pérdida de la vitamina B1 en alrededor } \\
\text { de un } 5 \% \text { con } 0,5 \mathrm{kGy} \text { en huevo seco } \\
\text { entero. } \\
\text { - Pérdidas insignificantes de vitamina } \\
\mathrm{C} \text { con hasta } 1 \mathrm{kGy} \text { en frutas frescas y } \\
\text { verduras }\end{array}$ & $\begin{array}{l}\text { Cocción (para conseguir el mismo } \\
\text { fin en frutas y verduras se pierde } \\
\text { considerablemente más vitamina C) }\end{array}$ & {$[21]$} \\
\hline Huesos & Radicales libres & & {$[21]$} \\
\hline
\end{tabular}

Fuente: autor

De los compuestos listados, varios aparecen en mayor proporción cuando se usan tratamientos convencionales que con dosis adecuadas de radiación, y otros, como los radicales libres, tienen tiempos de vida media muy cortos en medios acuosos, por lo que solo suelen permanecer en huesos y productos en estado congelado. En el caso de las 2-ACB, se encontró que se producen exclusivamente por radiación y que se deben realizar mayores estudios toxicológicos para verificar que su consumo es inocuo en los niveles producidos por la radiación, de modo tal que deben ser considerados un aditivo [20]. Si comparamos el efecto de la irradiación en vitaminas aisladas en solución con el efecto de irradiarlas dentro de la matriz propia del alimento, el daño es casi diez veces menor, pues parece existir un efecto protector de otros compuestos antioxidantes presentes de modo natural [21].

Son varios los beneficios que la técnica de irradiación ofrece, como el hecho de ser un proceso seguro y efectivo que contribuye a mantener la calidad e inocuidad de los alimentos [5], [17], rompe barreras cuarentenarias [23] y aumenta tiempos de vida útil [17], [24]. En contraste con las tecnologías convencionales, presenta una ventaja competitiva en los ámbitos comercial y de salubridad para el consumidor; para ello se debe validar cada caso antes de someter lotes enteros de material a tratamiento, razón por la cual existe la planta piloto de irradiación, sobre la que se hablará más adelante.

\section{APLICACIONES DEL TRATAMIENTO DE ALIMENTOS CON RADIACIÓN IONIZANTE}

Durante la irradiación se transfiere energía con carácter ionizante de una fuente de radiación, usualmente gamma, al producto tratado. El parámetro más importante del proceso de irradiación es la cantidad de energía ionizante absorbida por unidad de masa del material, denominada "dosis absorbida" o simplemente "dosis" [13].

Como tales, las diferentes aplicaciones dependen del fin último y de la dosis aplicada al alimento, y varía mucho entre productos, aunque se conocen dosis genéricas que se usan en procesos determinados. Las denominaciones que tienen los tratamientos desde años atrás, como radapertización, aplicada a los tratamientos de esterilización; radurización, aplicada a la descontaminación de bacterias que deterioran la calidad, con el fin de extender el tiempo de vida útil, o radicidación, usada para reducir cargas de parásitos y bacterias no esporuladas patógenas, como el gusano plano, la Trichinella, y la E. Coli o la Salmonella [25], ya no son de uso común, por lo que en este momento el Manual de buenas prácticas de irradiación del OIEA [13] clasifica los tipos de tratamiento simplemente a partir de las aplicaciones o fines de irradiación. 
Servicio Geológico Colombiano

Tabla 3. Aplicaciones de la radiación gamma en alimentos

\begin{tabular}{|c|c|c|c|}
\hline $\begin{array}{c}\text { Aplicación de la } \\
\text { irradiación en alimentos }\end{array}$ & Productos tratados & $\begin{array}{l}\text { Rango de dosis } \\
\text { (kGy) [6], [12] }\end{array}$ & $\begin{array}{l}\text { Nivel de la dosis suministrada } \\
\text { (bajo, medio, alto)[6] }\end{array}$ \\
\hline Inhibición de la germinación & Papa, batata, cebolla, ajo & $0,02-0,15$ & Bajo \\
\hline $\begin{array}{l}\text { Desinfestación de insectos } \\
\text { y seguridad cuarentenaria }\end{array}$ & $\begin{array}{l}\text { Granos, cereales, café, especias, } \\
\text { frutas, productos de pescado, nueces } \\
\text { deshidratadas, } \\
\text { mango y papaya. Algunas Frutas y } \\
\text { vegetales. }\end{array}$ & $0,02-1$ & Bajo \\
\hline Retraso de la madurez (radurización) & Papa, frambuesa & $0,01-3$ & Bajo \\
\hline $\begin{array}{l}\text { Inactivación de parásitos y bacterias } \\
\text { patógenas no esporuladas (radicidación) }\end{array}$ & $\begin{array}{l}\text { Carnes, nueces, cereales, especias, etc. } \\
\text { No deshidratados }\end{array}$ & $0,3-8$ & Medio \\
\hline $\begin{array}{l}\text { Reducción de las cargas microbianas } \\
\text { que causan deterioro, para extensión de } \\
\text { la vida útil }\end{array}$ & $\begin{array}{l}\text { Granos, cereales, especias, nueces, } \\
\text { deshidratados, } \\
\text { entre otros }\end{array}$ & $1-10$ & Medio \\
\hline Reducción de bacterias patógenas & $\begin{array}{l}\text { Ingredientes deshidratados (especias, } \\
\text { hierbas } \\
\text { y condimentos) }\end{array}$ & $1-20$ & Alto \\
\hline $\begin{array}{l}\text { Esterilización para extender el tiempo } \\
\text { de vida de los productos y hacerlos } \\
\text { estables a temperatura ambiente } \\
\text { (radapertizacion) }\end{array}$ & Carnes de res, cerdo y ave & $\begin{array}{l}>25 \text { (dependiendo del producto, la } \\
\text { esterilidad se puede dar a partir de } 10 \\
\text { kGy) }\end{array}$ & Alto \\
\hline
\end{tabular}

Fuente: autor

La irradiación gamma, al atacar los microbios e insectos, no solo ayuda a combatir las enfermedades causadas por patógenos, sino que es una manera efectiva de reducir las pérdidas debidas a la infestación, contaminación y deterioro de los alimentos [12], [25], que según datos de la FAO, llegan en este momento a afectar a casi un tercio de la producción global de alimentos [26].

\section{LA PLANTA DE IRRADIACIÓN GAMMA DEL SERVICIOGEOLÓGICO COLOMBIANO}

Desde comienzos de los años setenta del siglo pasado, el antiguo Instituto de Asuntos Nucleares (IAN) conocía las ventajas ya mencionadas de la técnica de irradiación para el tratamiento de alimentos. Tiempo atrás, en 1961, para ser precisos, se habían realizado los primeros experimentos de irradiación de papa para conservación a 100 Gy con la fuente de $850 \mathrm{Ci}$ de cobaltoterapia del Instituto de Cancerología (INC) [14].

A partir de 1968 el IAN, viendo la importancia que tiene la técnica para el sector agrícola del país, presentó al OIEA un proyecto de cooperación técnica en química de radiaciones, y obtuvo la aprobación para importar una fuente de $10000 \mathrm{Ci}$ proveniente de Estados Unidos. Para ello se constituyó un equipo de trabajo conformado por personal del Instituto, que emprendió el diseño de una instalación multipropósito que "sirviese para adelantar proyectos de puesta a punto y demostración tecnológica en todas las áreas de interés para la entidad y el país" [14]. En 1972 fue terminada la obra civil, y para 1976 la planta ya había sido dotada con los sistemas de dosimetría de alta dosis y sistemas auxiliares dentro de la piscina y con la fuente de ${ }^{60} \mathrm{Co}$; sin embargo, se realizarían reforzamientos en el blindaje biológico de concreto para soportar las recargas a 100000 Ci llevadas a cabo más adelante.

La Planta de Irradiación está clasificada en el nivel IV, de cuatro tipos de diseños de irradiadores de fuente gamma, y es categoría 1, la más alta según la Resolución 180052 de 2008 del Ministerio de Minas y Energía [27], dado que logra almacenar material radiactivo con una actividad total de $100000 \mathrm{Ci}$, cuya última recarga se dio en mayo de 2013. La radiación es producida por un isótopo del cobalto denominado ${ }^{60} \mathrm{Co}$, que está encapsulado en vainas de acero inoxidable tipo ${ }^{188} \mathrm{C}$, diseño de la compañía canadiense Nordion [7]. Este isótopo no se encuentra en la naturaleza, por lo que es producido en reactores nucleares a partir de la activación neutrónica del metal de cobalto-59, el cual se dispone en lápices o barras que son luego transportadas de manera especial hasta la facilidad, donde son dispuestas una a una en un marco portafuentes, tal que permita una distribución uniforme de la dosis de radiación del producto que se desplazará alrededor de dicha fuente. A diferencia de un 
acelerador de electrones, una fuente radiactiva no se puede apagar, y su radiación es continua. A medida que decae a níquel-60 emite dos rayos gamma de 1,17 y 1,33 MeV [25], $y$ tiene una vida media de 5,3 años.

Las fuentes son almacenadas en una piscina profunda que actúa como blindaje biológico, impidiendo que la radiación escape; por lo demás, no hay activación alguna del agua [25]. En comparación con otras tecnologías de irradiación, como los rayos $\mathrm{X}$ y los electrones acelerados, los rayos gamma tienen mayor penetrabilidad y su producción depende de un isótopo que decae naturalmente, y no de máquinas que consumen energía para su operación [25]. En la figura 1 se pueden observar las principales partes que componen la facilidad; para simplificar, en la misma no aparece el sistema de transporte de rodillo vivo que rodea el borde de la piscina de almacenamiento en la sala de irradiación.
Aunque la tecnología de irradiación ionizante ha demostrado ser un proceso seguro y un tratamiento efectivo de alimentos, son otras las aplicaciones de irradiación que se aprovechan en la planta del Servicio Geológico Colombiano, como el tratamiento con fines de esterilización de insumos médicos y quirúrgicos, y el tratamiento de materias primas para los sectores de cosmética y farmacia. Pero son muchas más las aplicaciones que se le pueden dar a una instalación de este tipo; entre ellas se encuentra el uso de la radiación como catalizador en reacciones de polimerización [28], conservación del patrimonio cultural [29], tratamiento de aguas residuales [30], mutaciones benéficas de especies vegetales [31] y técnica del macho estéril para control de moscas de la fruta [32], [33].

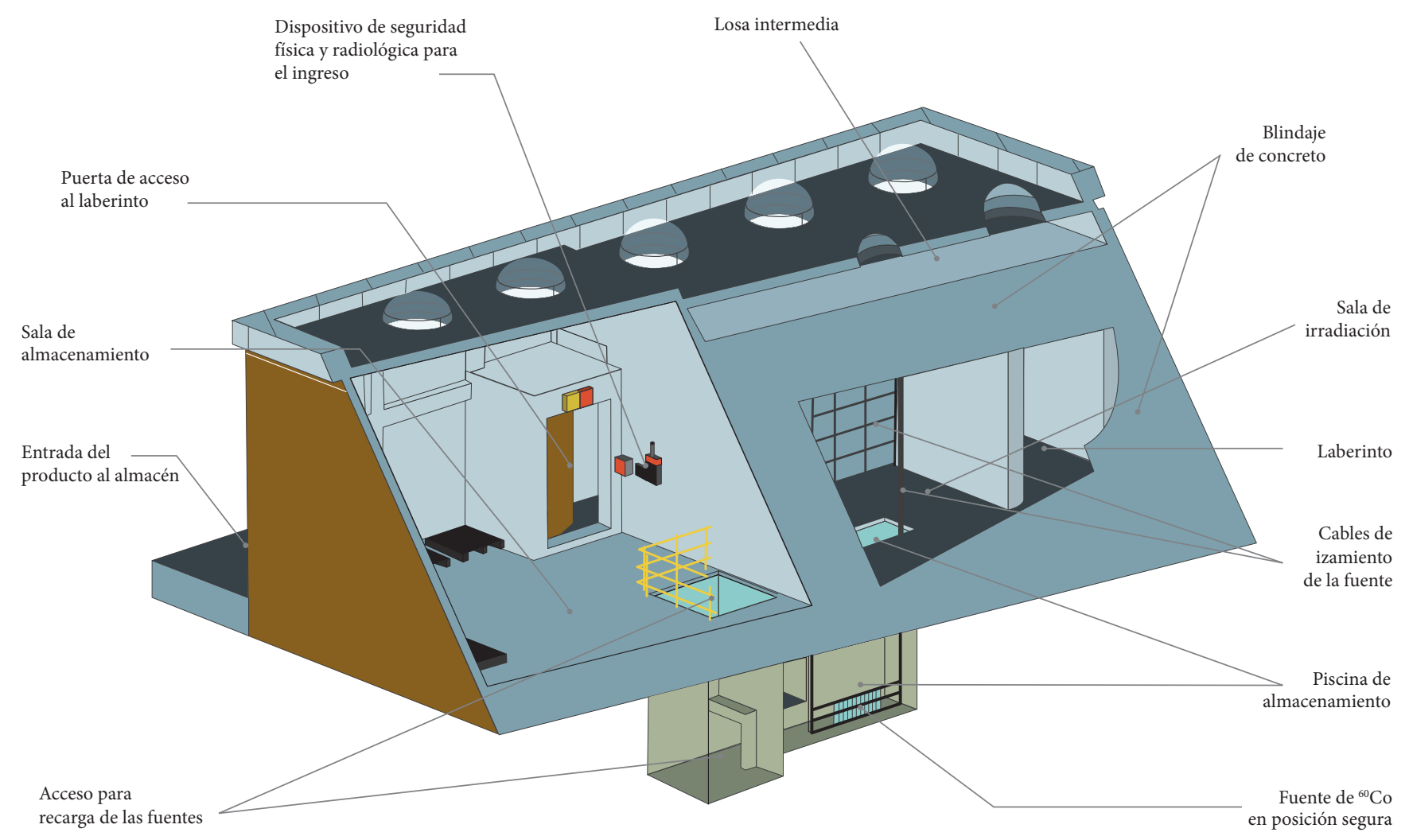

Figura 1. Vista en corte transversal de la Planta de Irradiación Gamma del SGC. Se identifican sus partes principales.

Fuente: autor 


\section{TRABAJOS DE INVESTIGACIÓN Y DESARROLLO TECNOLÓGICO REALIZADO EN LA PLANTA DE IRRADIACIÓN}

La siguiente tabla menciona algunos de los trabajos que se han llevado a cabo en la Planta de Irradiación Gamma. No todos se encuentran, y muchos de ellos no están disponi- bles, pero es un punto de partida para entender que se deben enfocar esfuerzos en proyectos de interés nacional y regional, de la misma forma que se investigan los efectos de la irradiación en alimentos en países en vías de desarrollo, donde ya se ha entendido que esta técnica les permite imponerse en el mercado internacional.

Tabla 4. Algunos trabajos de investigación llevados a cabo en la Planta de Irradiación del SGC

\begin{tabular}{|c|c|c|c|}
\hline Trabajo de investigación & Instituciones participantes & Resultados & Referencia \\
\hline $\begin{array}{l}\text { Consideraciones sobre la viabilidad } \\
\text { de la construcción de una facilidad } \\
\text { gamma para operación comercial en la } \\
\text { República de Colombia (ST-DRT-03-92). } \\
\text { Anexo técnico sobre des infestación de } \\
\text { frutas }\end{array}$ & IAN & Desconocidos para el autor & {$[14]$} \\
\hline $\begin{array}{l}\text { Aplicación de la técnica de radiación } \\
\text { ionizante como tratamiento } \\
\text { cuarentenario de frutas de exportación }\end{array}$ & ICA, Ingeominas, Asohofrucol & $\begin{array}{l}\text { Se validó el uso de radiación } \\
\text { ionizante gamma como tratamiento } \\
\text { cuarentenario en doce frutas de interés } \\
\text { para el mercado de EE. UU.: tomate de } \\
\text { árbol, mangostino, uchuva, pitahaya, } \\
\text { granadilla, maracuyá, feijoa, lulo, } \\
\text { papaya hawaiana, curuba y gulupa. Sin } \\
\text { respuestas adversas ni cambio en las } \\
\text { propiedades organolépticas }\end{array}$ & {$[14],[34]$} \\
\hline $\begin{array}{l}\text { Mutagénesis y mejoramiento genético } \\
\text { del arroz, y genómica funcional en el } \\
\text { mejoramiento del arroz }\end{array}$ & UD, Ingeominas & $\begin{array}{l}\text { Se validó el uso de radiación ionizante } \\
\text { gamma para la inducción controlada de } \\
\text { mutaciones en especies de arroz }\end{array}$ & {$[35]$} \\
\hline $\begin{array}{l}\text { Efecto del tratamiento de irradiación en } \\
\text { la calidad de albahaca, menta y romero, } \\
\text { en poscosecha }\end{array}$ & U. NAL., Ingeominas & $\begin{array}{l}\text { Se validó el uso de radiación ionizante } \\
\text { gamma para el control de plagas en } \\
\text { poscosecha en plantas aromáticas de } \\
\text { exportación }\end{array}$ & {$[14],[36]$} \\
\hline $\begin{array}{l}\text { Physicochemical Properties of Oils } \\
\text { Extracted from c-Irradiated Sacha Inchi } \\
\text { (Plukenetia volubilis L.) Seeds }\end{array}$ & U. NAL., SGC & $\begin{array}{l}\text { Se irradiaron semillas de sacha inchi a } \\
1,5 \text { y } 8 \text { kGy, con una disminución no } \\
\text { significativa de los tocoferoles presentes } \\
\text { en el aceite y un aumento también } \\
\text { no significativo de los productos de } \\
\text { oxidación lipídica. Se demuestra que la } \\
\text { irradiación es un tratamiento adecuado } \\
\text { y ecológico para la desinfestación y } \\
\text { conservación de esta semilla }\end{array}$ & [37], [38] \\
\hline
\end{tabular}

Fuente: autor

\section{CONCLUSIONES}

La irradiación gamma en aplicaciones alimentarias ha demostrado su efectividad, a pesar de las controversias que surgen de su implementación por el posible rechazo de los consumidores, dada su tendencia radiófoba hacia las técnicas nucleares en general. Sin embargo, su uso ha sido aprobado por la regulación de alrededor de cincuenta países del globo, debido a que son pocos los efectos negativos sobre la composición y el poder nutricional de los alimentos, lo que hace que los efectos de este tratamiento sean comparables a los del tratamiento térmico, en el que la cantidad de ener- gía impartida, sea en forma de calor o radiación ionizante, puede causar efectos negativos, si se exceden los valores prestablecidos.

En Colombia solo contamos con el irradiador del SGC para llevar a cabo investigación y prestación de servicios, pues la transferencia tecnológica al sector privado pensada años atrás quedó rezagada por decisiones políticas de finales del siglo pasado. Por eso, conviene que tanto el sector público como el privado reconozcan que el uso pacífico de las tecnologías radiactivas y nucleares es una pieza clave para alcanzar niveles adecuados de desarrollo en las diferentes áreas del conocimiento. También es necesario que las uni- 
versidades incrementen los proyectos de investigación con miras a fortalecer los pilares del desarrollo tecnológico nuclear que el país necesita.

En perspectiva, se nota una tendencia a retomar la irradiación de alimentos como alternativa a diversos tratamientos convencionales que representan mayores costos, o ante la imposibilidad de conseguir los mismos resultados con otros procedimientos. Esta tecnología radiactiva resulta atractiva para los países en vías de desarrollo, que acrecientan su conocimiento en la materia con los programas de cooperación regional y nacional del OIEA que permanecen activos.

Dada la última recarga realizada a la Planta de Irradiación del SGC en 2013, a la fecha se cuenta con aproximadamente $60000 \mathrm{Ci}$, que, para fines de investigación, es una actividad lo suficientemente grande para alcanzar las dosis en muestras de varios volúmenes y en tiempos más cortos que los obtenidos con un irradiador de una actividad inferior para la misma dosis, como aquellos usados en otras aplicaciones. En consecuencia, se deduce que existe un potencial para retomar el trabajo de investigación en todas las áreas en que la Planta de Irradiación pueda contribuir al desarrollo tecnológico del país, tanto en el sector académico como en el de la industria privada.

\section{Referencias}

[1] M. Lacroix y B. Ouattara, "Combined Industrial Processes with Irradiation to Assure Innocuity and Preservation of Food Products: A Review", Food Res. Int., vol. 33, n. ${ }^{\circ}$ 9, 2000, pp. 719-724.

[2] M. K. Dogbevi, C. Vachon y M. Lacroix, "Effect of Gamma Irradiation on the Microbiological Quality and on the Functional Properties of Proteins in Dry Red Kidney Beans (Phaseolus vulgaris)", Radiat. Phys. Chem., vol. 57, 2000, pp. 265-268.

[3] U. Gecgel, T. Gumus, M. Tasan, O. Daglioglu y M. Arici, "Determination of Fatty Acid Composition of g-Irradiated Hazelnuts, Walnuts, Almonds, and Pistachios", Radiat. Phys. Chem., vol. 80, n. ${ }^{\circ} 4$, 2011, pp. 578-581.

[4] M. Arici, F. Arslan Colak y Ü. Gecgel, "Effect of Gamma Radiation on Microbiological and Oil Properties of Black Cumin (Nigella sativa L.)", Grasas y Aceites, vol. 58, n. ${ }^{\circ}$ 4, 2007, pp. 339-343.
[5] R. A. Molins, Y. Motarjemi y F. K. Käferstein, "Irradiation: A Critical Control Point in Ensuring the Microbiological Safety of Raw Foods", Food Control, vol. 12, 2001, pp. 347-356.

[6] X. Fan and C. H. Sommers, Eds., Food irradiation research and technology, Second. IFT press, 2012.

[7] P. Kunstadt, "Food Irradiation: Gamma Processing Facilities", en Seminario National de Irradiacidn de Alimentos, 1997, pp. 47-64.

[8] D. L. Alonso, V. M. Pabón, G. A. Parrado y J. C. Parada, "Revisión sobre la producción de radionúclidos en reactores nucleares y sus aplicaciones como radiotrazadores", Investig. y Apl. Nucl., n. ${ }^{\circ}$ 1, 2017, pp. 6-23.

[9] S. Henson, "Demand-Side Constraints on the Introduction of New Food Technologies: The Case of Food Irradiation", Food Policy, vol. 20, n. ${ }^{\circ} 2$, 1995, pp. 111-127.

[10] P. B. Roberts, "Food Irradiation Is Safe: Half a Century of Studies", Radiat. Phys. Chem., vol. 105, 2014, pp. 78-82.

[11]D. A. E. Ehlermann, "The Early History of Food Irradiation", Radiat. Phys. Chem., vol. 129, 2016, pp. 10-12.

[12]J. Farkas y C. Mohácsi-Farkas, "History and Future of Food Irradiation", Trends Food Sci. Technol., vol. 22, n. ${ }^{\text {os }}$ 2-3, 2011, pp. 121-126.

[13] International Atomic Energy Agency (IAEA), "Manual of Good Practice in Food Irradiation: Sanitary, Phytosanitary and Other Applications", Tech. Reports Ser., n. ${ }^{\circ}$ 481, 2015, pp. 1-85.

[14]H. A. Múnera, "La Planta de Irradiacion Gamma", en Tecnología Nuclear No Médica en Colombia. Pasado, presente y futuro, BOGOTÁ, D.C.: Tecnicontrol S.A., 2011, pp. 167-188.

[15] S. F. Sabato, J. M. Silva, J. N. Cruz et al., "Advances in Commercial Application of Gamma Radiation in Tropical Fruits at Brazil", Radiat. Phys. Chem., vol. 78, n. ${ }^{\text {ss }} 7-8$, 2009, pp. 655-658.

[16]Z. Lakner, S. Soós, Z. Vida y C. Farkas, "European Research and the Hungarian School of Food Irradiation", Radiat. Phys. Chem., vol. 129, 2016, pp. 13-23.

[17]L. M. Crawford y E. H. Ruff, "A Review of the Safety of Cold Pasteurization Through Irradiation", Food Control, vol. 7, n. ${ }^{\circ}$ 2, abr. 1996, pp. 87-97.

[18]G. R. Malkoske, "Total Quality Management of Cobalt-60 Sources", Radiat. Phys. Chem., vol. 54, n. ${ }^{\circ}$ 6, 1999, pp. 601-608. 
[19] A. Prakash, "Chapter 17 Irradiation of Nuts", en Food Irradiation Research and Technology; Second. X. Fan y C. H. Sommers, eds. Orange, USA: Blackwell Publishing and the Isntitute of Food Technologists, 2013.

[20] H. Sommers, C. H. Delincée, J. S. Smith y E. Marchioni, “Toxicological Safety of Irradiated Foods", en Food Irradiation: Research and Technology, 2012, pp. 53-73.

[21] Organización Mundial de la Salud, Inocuidad e idoneidad nutricional de los alimentos irradiados, Ginebra: Organización Mundial de la Salud, 1995.

[22] M. Carocho, P. Morales e I. C. F. R. Ferreira, "Natural Food Additives: Quo Vadis?”, Trends Food Sci. Technol., vol. 45, n. $^{\circ} 2,2015$, pp. 284-295.

[23] I. S. Arvanitoyannis y P. Tserkezou, Legislation on Food Irradiation: European Union, United States, Canada, and Australia, London: Elsevier Inc., 2010.

[24] I. Ihsanullah y A. Rashid, "Current Activities in Food Irradiation as a Sanitary and Phytosanitary Treatment in the Asia and the Pacific Region and a Comparison with Advanced Countries”, Food Control, vol. 72, 2017, pp. 345-359.

[25] L. McKeen, Introduction to Food Irradiation and Medical Sterilization. 2012.

[26]FAO, "Pérdida y desperdicio de alimentos", 2018. [Online]. Disponible en http://www.fao.org/food-loss-andfood-waste/es/. [Consultado: 2-jul.-2018].

[27] Ministerio de Minas y Energía, Sistema de categorización de las fuentes radiactivas, vol. 18 0052, Colombia, 2008, p. 12.

[28] V. H. Poblete, M. Álvarez y M. E. Pilleux, "Estudio exploratorio de las propiedades eléctricas de nanocompositos de cobre-PMMA, polimerizados mediante radiación gamma”, Nucleotecnica, vol. 36, no. Diciembre, p. $27,2002$.

[29] M. Maria, C. D. Negut, I. Rodica y C. C. Ponta, "Irradiation Effects on Canvas Oil Painting: Spectroscopic Observations”, Radiat. Phys. Chem., vol. 81, n. ${ }^{\circ}$ 10, 2012, pp. 1595-1599.

[30]Y. Wu, Y. Jiang, G. Ke y Y. Liu, "Effect of Gamma-Ray Irradiation on the Dewaterability of Waste Activated Sludge", Radiat. Phys. Chem., vol. 130, 2017, pp. 164-170.
[31]K. Ulukapi y S. Fatih, "Study of the Effect of Irradiation (60 Co) on M 1 Plants of Common Bean (Phaseolus vulgaris L.) Cultivars and Determined of Proper Doses for Mutation Breeding”, J. Radiat. Res. Appl. Sci., vol. 11, n. ${ }^{\circ}$ 2, 2018, pp. 157-161.

[32] G. J. Hallman y P. Loaharanu, "Phytosanitary Irradiation: Development and Application", Radiat. Phys. Chem., vol. 129, 2016, pp. 39-45.

[33] G. J. Hallman, Y. M. Hénon, A. G. Parker y C. M. Blackburn, "Introductory Papers Phytosanitary Irradiation: An Overview”, Florida Entomol., vol. 99, n. ${ }^{\circ}$ Special Issue 2, 2016, pp. 1-13.

[34] G. M. Vidal, R. E. Orjuela y A. Moreno, "Aplicación de la técnica de radiación ionizante como tratamiento cuarentenario a frutas de exportación, Convenio ICA-Asohofrucol (FNHF)-Ingeominas (SGC)", en Seminario Taller de Irradiación Fitosanitaria, 2016.

[35] J. O. Quevedo, L. A. OSPINA y J. H. Beltrán, “Efectos de las radiaciones gamma sobre la capacidad androgénica de seis genotipos de arroz (Oryza sativa 1.)", Asquimco, vol. 30, n. ${ }^{\circ} 82,2008$.

[36]L. Patiño y A. Herrera, "Aplican tecnología limpia a poscosecha de albahaca, menta, tomillo y romero", Agencia de Noticias UN, 2015. [Online]. Disponible en http:// agenciadenoticias.unal.edu.co/detalle/article/aplican-tecnologia-limpia-a-poscosecha-de-albahaca-menta-tomillo-y-romero.html. [Conslultado: 2-jul.-2018].

[37]L. F. Gutiérrez, Y. Quiñones-Segura, Z. Sánchez-Reinoso, D. L. Díaz y J. I. Abril, "Physicochemical Properties of Oils Extracted From $\gamma$-Irradiated Sacha Inchi (Plukenetia volubilis L.) Seeds", Food Chem., vol. 237, 2017, pp. 581-587.

[38]L. Gutiérrez y J. Abril, "Irradiación gamma no afecta aceite de semilla amazónica”, Agencia de Noticias UN, 2017. [Online]. Disponible en http://agenciadenoticias. unal.edu.co/detalle/article/irradiacion-gamma-no-afecta-aceite-de-semilla-amazonica.html. 